TM-1574

\title{
Report of the Ad Hoc Committee on Test Beam Policy
}

Committee Members:

R. Stefanski (Chair), D. Anderson, S. Childress, R. Coleman, H. E. Fisk, P. Garbincius, S. Holmes, R. Kephart, M. Kreisler, H. Lubatti, and J. Siegrist Fermi National Accelerator Laboratory

P.O. Box 500, Batavia, Illinois

March 20, 1989 


\section{Report of the Ad Hoc Committee on Test Beam Policy}

Committee Members:
D. Anderson
S. Childress
R. Coleman
H.E. Fisk
P. Garbincius
S. Holmes
R. Kephart
M. Kreisler
H. Lubatti
J. Siegrist
R. Stefanski, Chair

\section{Recommendations of the Committee}

1. Purpose

This document was developed at the request of the Physics Advisory Committee of the Fermi National Accelerator Laboratory to "review the general subject of test beams with the purpose of establishing general policy and guidelines for consideration of future test beam requests".

The recommendations stated here should be subject to periodic review, since the Laboratory position must change as needs and available resources change.

\section{Recognition of Need}

\subsection{Criteria for Determining Need}

Fermilab should consider seriously any request for test beam time from any group.

In general, experiments approved at Fermilab must request test beam needs as part of the approval process or must submit supplemental requests to the management if needs change. As is Laboratory policy for all experiments, the Laboratory will require an adequate understanding of the funding of the effort before test beam time is allocated.

Fermilab should also consider requests by groups conducting experiments or projects at another laboratory or as part of SSC R\&D programs. It should be Fermilab policy to resolve conflicts among users during fixed target running periods according to priorities set by the Director of Fermilab with guidance from the PAC.

As an example, experiments to be carried out at Fermilab should state their test beam needs as part of the review process, and approval should be contingent on a commitment by all parties to identify funds and resources for test beam activities as required. E-740 (D0) and E-741 (CDF) are examples of such needs. Future experiments of this magnitude should be required to state their needs before phase II approval is given, and approval for test beam use should be limited in duration.

An experiment or project to be carried out at an outside laboratory can represent a need for test beam at Fermilab provided the funding agency recognizes and accepts the costs associated with the project's activities at Fermilab. 
Fermilab policy should be to recognize and fulfill,to the best of its ability, all approved needs as defined in this section. When conflicts arise among major (See section 1.2.3) test beam users and the fixed target physics program, the Laboratory Director should seek advice from the PAC on methods to resolve the conflict.

\subsection{Priority Among Needs}

The Laboratory policy will be to meet its commitments to all users. Priorities are used for decisions involving short term allocation of resources. As such, priorities are to be assigned by the PAC for major test beam users even as they are for running experiments.

This committee makes no specific recommendation for assignment of priorities among the currently recognized test beam needs. Because the fixed target program runs infrequently, it is clear that prudent management of resources is essential to meet all of the program commitments.

\subsection{Distinction Between Major and Minor Users}

A request for test beam that does not create a conflict of Laboratory resources, and does not conflict with the ongoing physics program and its schedule, will be considered a minor need. The decision to fulfill these needs should be based on the criteria given in paragraph 2.1, and should be carried out by the Program Planning Office. A minor need may not require a review by the PAC.

A Laboratory representative should be named to act as liaison for minor test beam users. Many test beam requirements for minor users may be handled directly by the Liaison Physicist, with appropriate consultation with Laboratory Management. The Liaison Physicist would also serve as spokes-person for minor test beam users, especially if existing facilities are too limited for the perceived needs.

\section{Availability Of Resources}

\subsection{Existing Test Beams}

The existing test beams at Fermilab are: MTest (CDF), MBottom, NWest (D0), and NT/NH (E-790). A review of their properties and the program for the ' 89 running period is given in Appendix C. The committee perceives that these beams will be fully utilized during the ' 89 run.

\subsection{Expansion of Test Beam Facilities}

In general Fermilab should discourage the addition of new beam lines to the Fixed Target Program for test beams. The present collection of beams is already very extensive and at times difficult to operate. It is likely that an addition of beams to this collection would merely reduce the operating efficiency of the exiting beams. The more pressing need is to develop test beam capabilities that would be available all year round.Phase II of the Fermilab Upgrade plan would provide this capability. 
If circumstances dictate that additional beams must be built in the fixed target areas, the expansion of test beam facilities should involve making greater use of existing beams. There are five opportunities for expansion, and these are listed in Appendix D. Fermilab should leave it to the potential new users, working with the Laboratory, to determine the approach best suited to their needs and the manner in which funding for the project could be obtained.

\subsection{Special Facilities}

A typical test beam user may require a variety of particle species and beam energies. The dynamic range needed by some users is not always possible to achieve at a single beam. Some groups travel to BNL or SLAC to carry out low energy tests, or tests in electron beams. Among our current major users, CDF does the best it can at low energy in MT; They prefer to do all of their tests in the same beam line.

There is also a need to study D0 calorimeter response with low energy hadrons. With the complications expected with a $10^{\prime}$ dia. by $20^{\prime}$ long cryostat in NWA, it is probably impractical to do anything other than provide a low energy test beam to NWA. The time scale for development of such a facility is about two years, since its need is not foreseen in the next fixed target run (Dec 1989). There is a small probability low energy testing for D0 could be done at BNL, but this involves duplicating cryogenic and data acquisition facilities there.

Another special facility that is often needed is a high quality electron beam (See E798). Many Fermilab groups have in the past traveled to SLAC to carry out careful lead glass studies. Electron test beams at Fermilab tend to be relatively pure at low energy $(<10 \mathrm{GeV})$, but are composed mainly of pions above about $25 \mathrm{GeV}$. In either case the electrons must be tagged. The major test beam users in NW and MT find their electron content and tagging systems adequate for their requirements. An improved facility for studies that require a high energy, relatively pure beam, could be done at the Wide Band Hall. A facility of this type would require minor modifications to the electron beam dump, but would not be available until E-774 completes its run.

The Laboratory should carry out a feasibility study for these special facilities, to determine if they could be developed with minimal expenditure of resources, and without interference with the physics program.

\subsection{Test Beams at Other Labs}

A survey was taken of the availability of test beams at BNL and SLAC. (See Appendix E ) The facility at BNL appears oversubscribed, and SLAC has not run a test beam in 2.5 years. BNL plans a 16 week running period in ' 89 , while SLAC may run for four weeks. These are low energy facilities which at times have proven invaluable for Fermilab groups. The Laboratory should encourage the continued use of BNL and SLAC for test beam activities when practical. 


\subsection{A Year-Round Program}

The most restrictive element in test beams available to the US High Energy Physics program is the limited time test beams operate. SLAC operates for 4 weeks a year at most, BNL seems to have a fifteen to twenty week operating period per year, and Fermilab operates fixed targets an average of 14 weeks a year. The best hope for improving test beam availability is to develop a year-round facility.

Furthermore, the single most promising possibility for an expansion of test beams at Fermilab would be to provide for extraction from the proposed new main injector, to the Fixed Target Areas during collider runs. This would effectively double the available test beam time.

Also,the availability of protons to the fixed target areas during collider runs will more than double the available test beams. For example, in addition to the standard test beams such as MT, MB, NW, NT, and NH, beams could be developed to take advantage of space in the Muon Lab and ME. An electron beam could be made available in Wide Band Hall also.

Fermilab should make every effort to develop a means to deliver protons to the fixed targets during collider runs.

Furthermore, a test beam area could be developed in conjunction with the new main injector. This area could reside at the Southwest corner of the site, and contain three or more test beams that operate all year round. The committee recommends that a design study be carried out for such an area.

\section{Concerns of the Fixed Target Physics Program}

The use of beam lines for tests of collider components is in direct competition with the needs of experiments in the fixed target program. As it happens, current test beams are those for which there exists no reasonable fixed target physics user. The beam energies are too low in MT, MB, and NW to be useful for a fixed target experiment, and the beam intensities are too low in NT (E-782 being a special case.). However, an increase in the demand for test beam space may require that a beam currently used for fixed target physics be converted to a test beam, or that new beams be constructed as test beams. The committee recommends that any proposal for such action be subject to PAC review, even as fixed target proposals are subject to such review. That is that a request for such action be accompanied by a proposal, and presentation, requiring PAC approval before the action is implemented. Furthermore, allocation of resources to test beams should not be considered permanent; Proposals may be considered by the PAC for fixed target experiments that would call upon the termination of a test beam to install the approved experiment. 
5 Summary and Recommendations

The committee makes the following recommendations:

Fermilab should cooperate with all test beam users to identify needs and sources of support not always assumed to come from Fermilab.

The committee makes no recommendation with regard to priority of test beam users, but reaffirms the role of the PAC to establish priorities among experiments and test beam activities. However, because of the short duration of the run, the ' 89 fixed target run must fulfill the needs of the fixed target experiments and the requirements of all recognized test beam needs.

The committee recommends that a feasibility study be carried out for special facilities such as low energy beams and relatively pure electron beams. The committee recommends the continued use of BNL and SLAC test beams when practical.

The committee is reluctant to recommend the expansion of existing fixed target facilities to provide additional test beams. Rather, the committee recommends that a means be developed to operate the fixed target beams during collider runs, to increase substantially the utility of these facilities. If a new beam must be built in the fixed target areas, the development of the new site should be worked out between the prospective new user and the Laboratory.

Commensurate with the need to develop year round facilities, the committee recommends the development of a conceptual design for test beams at the new main injector, proposed to be built in 1993.

Finally, the Committee recommends that the PAC act as the agent for review of test beam facilities when they are in conflict with fixed target experiments. 
Appendices

Appendix A

Status of the $120 \mathrm{GeV}$ extraction to the Fixed Target Areas

Test beams are unavailable when the fixed target program is not running. The greatest opportunity for increasing the availability of test beams is to make such beams available during these collider and accelerator studies times. The new main injector may offer an opportunity to do so by providing $120 \mathrm{GeV}$ protons to a new test beam area or to the fixed target program. This beam, under the present proposed schedule, would not be available until 1993. If $120 \mathrm{GeV}$ protons are delivered to the fixed target areas, the existing fixed target beam lines could be used to deliver particles to test beam areas. This would greatly increase the utility of these facilities for test beams.

The Accelerator Division is involved in developing a means for extraction of 150 $\mathrm{GeV}$ protons to the fixed target areas during collider runs before the construction of the new injector. This approach, which involves using the existing main ring, has some serious problems. The problems are associated with backgrounds at collider experiments during extraction, and the limited aperture for extraction in the ring. Tests may be carried out during the present collider run, but this approach seems unlikely to succeed. 
Appendix B

Projected Test Beam Requirements

B.1. Current and Future Test Beam Needs

The presently recognized needs for test beam at Fermilab are E-740 (D0), E-741 (CDF), E-790 (ZEUS), P-795 (SSC Warm Liquid R\&D), and P-798 (Synchrotron Radiation Detector).

P.797 (Small Diameter, Fast-Drift Proportional Tubes) is a new request for test beam.

The anticipated future needs for test beam are as follows:

1.Additional new SSC R\&D projects as stated in Appendix B.5.

2. Small R\&D users. See Section 2.3 and Appendix B.7.

3. Bottom Collider Detector

\section{B.2. $\mathrm{CDF}$}

CDF will want a test beam for essentially all of the fixed target runs for the foreseeable future. In practice they plan on using MT with "first priority" but coexisting with other users with whom they are compatible and who might consume up to $25 \%$ of the beam time. Their plan is to recalibrate wedges, plug, Fwd calorimetry plus misc tests associated with their upgrade plans.

Their beam needs are

50-250 GeV hadrons

$$
\text { 25-125 GeV electrons }
$$

In addition electrons and hadrons in the range 1-25 GeV are desirable but probably the experiment wouldn't move to another beam to get them. (A plan is in the works to modify MT to make this possible but the space required might be that otherwise occupied by other potential users.) The low energy beam design is being developed as a modification of a design originally done for $\mathrm{D} 0$ in $\mathrm{NW}$.

B.3. D0

$\mathrm{D} 0$ is in the process of installing a large cryogenic facility for testing various loads of calorimeter modules. The test vessel is sized to take their largest modules, namely the end calorimeter electromagnetic and inner hadronic detectors (40T). Their schedule is tight since they need to test one of the EC, EM, and IH detectors between the beginning and middle of the next fixed target run. The aim of their studies is calibration at the half percent level or better. To succeed, they need to understand the losses of energy deposited in between various calorimeter modules.

The beam needs are similar to those of CDF: hadrons and electrons between 5 and $150 \mathrm{GeV}$.

Their program is so extensive that it is unlikely that other users should plan to use this area. They are doing tests of their central tracking chambers that will continue into the next fixed target run. 


\section{B.4. Bottom Collider Detector}

The Bottom Collider Detector (BCD) will need test beam mainly before the detector is built. Unlike CDF and D0, the BCD has no hadron calorimetry and will therefore not require that modules be brought back for calibration after the detector is built. During detector development and construction the BCD detector group would require a test beam of well tagged pions, kaons, electrons and protons with energies between 0.5 and $50 \mathrm{GeV}$.

Since $B C D$ is now an approved $R \& D$ project , this group will need a test beam in the ' 89 run. It will be a challenge to find a place for them along with the SSC and other Tevatron collider requests.

\section{B.5. SSC}

SSC R\&D will likely proceed through several stages.

First, the exploration of new detector ideas for SSC. This is mostly what the Committee on SSC R\&D has funded so far. Many of these will need only minimal test beam time.

For this first stage, if we accept P-795 as an example of a near term SSC related test beam request, we would conclude that they are likely to be modest in size and beam time requirements. P-795 needs 600 hours of beam and floor space that measures $20 \mathrm{ft}$ in length by $10 \mathrm{ft}$ in width. Even, with such modest requests, however, we find that it is difficult to find room for them in the crowded fixed target areas. These requests would be easy to fulfill if protons were available during the collider run.

Second, the execution of prototype tests of near full scale detector components. (Especially calorimeters, and some silicon tracking devices.) The Committee on SSC R\&D has funded several such tests but many more are likely to be on the way. These will make substantial requests for test beam support. The calorimeter tests will want high energy electron and hadron test beams and will need substantial amounts of space. The Fixed Target run after the 1990 collider run would be about the right time scale for these requests, but some people will not be happy about waiting that long. Furthermore, as mentioned in section B.8, Fermilab and Argonne may take on major responsibility for the construction of SSC detectors, and, as such, become major test beam users at Fermilab.

Third, the execution of prototypes of detectors for an approved SSC detector. Extensive test beam programs for each large experiment will be required. The UA1, $\mathrm{UA2}, \mathrm{CDF}$ and $\mathrm{D} 0$ experience is ample evidence that such resources will be required. Moreover, these tests if done at FNAL should not be tied to the FT/Collider cycle but rather should provide more or less continuous access to this resource. Test beams should be provided from the injector at the SSC or from the proposed new Main Injector at FNAL.

Finally, an accurate projection of SSC requirements for tests beam cannot be made with currently available knowledge. The CDG will know more about short term needs after the November meeting of the SSC R\&D review panel. Additional study may take the form of a study group organized by the CDG, and consisting of representatives from major laboratories, to better coordinate test beam use for the SSC. The issues related to the SSC will evolve with time, and very likely change as the new facility is created. The fear is that we may fail to provide adequate facilities 
for the SSC. Nevertheless, the initiative for developing a long term plan must rest with SSC management. Fermilab's role must be to stand ready to assist the SSC management in every way possible, and a mechanism must be found to involve both laboratories in the planning process for these facilities.

\section{B.6. Fixed Target Users}

An examination of many of the fixed target programs shows that most experiments would profit from the existence of particles in their beam lines during the entire year. When a new piece of a spectrometer is added, when an old device is repaired, when readout electronics is being modified, a small amount of beam can determine if the changes are successful -- at a fraction of the time and cost of using the first two months of any fixed target running cycle for the " shakedown " phase.

Of course, one does not need to be too imaginative to realize that calibration of detector elements in situ could and would be made if such beams were available. Also, the benefits derived from the ability to train students during the collider running period could be considerable.

Such test beam in the fixed target beam lines could be scheduled to illuminate different beam lines at different times, could be at low energy and low intensity, etc. However, the general gist of many conversations on this topic is that there is a great deal of interest from fixed target experiments in the existence of beam at their detectors during the year of collider running.

This set of users represents a community that is rather large, and the cost effectiveness of such an option is favorable (especially when judged in terms of improving the efficient use of fixed target beam time).

\section{B.7. Small R\&D Efforts}

The fixed target areas have had only two small R\&D users in the last three running periods. These were tests carried out by Notre Dame on scintillating fibers for E687 , and streamer chamber tests for T-755, now part of E-791. Other tests have been carried out by Fermilab groups at BNL. These users are few and have very modest requirements in floor-space and time. As with P-795, finding nooks and crannies for these users is becoming difficult because of the full fixed target program. The scintillating fiber target was tested in NT, and T-755 was done downstream of CDF in MT.

An example of small R\&D requirements for test beams was given by David Anderson of the Particle Detector Group: Their main problem with test beams involves the difficulty in getting into a beam during Fermilab collider runs. The average wait of twenty months between fixed target runs isn't acceptable for a R\&D effort. On average, this group uses a test beam for about four weeks a year with large variations between years. Most of their test beam work involves calorimetry. The availability of protons from a main injector would be a great help for this group; Protons from the Booster would not be useful for their work. 


\section{B.8 Tests for Major Detectors at Other Laboratories}

American universities are involved in experiments at all major world laboratories including HERA, CERN, KEK, and UNK. Test beam activity at Fermilab is useful for such collaborations if some major portion of the detector is being built in the US and near Fermilab. Since a major part of the ZEUS detector is being built at ANL, this group will test their modules at Fermilab.

The degree to which this source of test beam user will be active in the future isn't clear. The case of having a portion of a detector built in the mid-west near Fermilab for an experiment in another country is somewhat special. However, unless challenging opportunities for physics are developed in the US during SSC construction, as would occur if the Tevatron Upgrade goes ahead, more US experimenters may work at experiments abroad, creating additional test beam needs of this kind.

In addition, both ANL and Fermilab may become major parties in the construction of SSC detectors, and, as such, may develop into a very significant test beam need from this source. 
Appendix C

Description of Existing Test Beam Facilities

Fermilab presently has four test beam areas: MTest, MBottom, NWest, and NTest. The allocation of time is as follows:

MTest is the E-741 (CDF) test beam. T-755 ran parasitically in this beam in the ' 87 run. In the ' 89 run, E-795 (Pripstein) will be in this beam also, but CDF will require the large majority of beam time.

MBottom cannot operate concurrently with MCenter, because of muon radiation in the pit. This is perhaps the best location for E-798(Russack) and P-797(Thun) in the ' 89 run, because MCenter (E-773) will be off for about half the run. However, several magnets must be installed in MBottom for the next run.

NWest is the test beam for E-740 (D0). They will have a substantial program for the ' 89 run that will require timely completion if the D0 detector is to be ready for beam in the ' 90 collider run. There is little chance for additional user's in this beam in the ' 89 run.

NTest will be used to complete E-782 in the ' 89 run and for E-790 test beam needs. This facility will be fully utilized in the ' 89 run. 
Appendix D

Description of an Expansion of Test Beam Facilities

\section{D.1 Potential for New Facilities}

Because space is limited during fixed target operation, and because $120 \mathrm{GeV}$ protons may not be available from a new injector until 1993 or later, the issue of adding new beams must be addressed. However, the ensemble of beams in the fixed target areas is already very complex and sometimes difficult to operate. One can't help but suspect that adding to these facilities will only reduce the operating efficiency of existing beams. Nevertheless, here are a few possibilities:

After the ' 89 running period, when E-782 is complete, the NT, NH, NK beams could be configured to share the slow spill by switching beam between them. In this way, three test beam users could be accommodated at a time, although with reduced duty cycle. If the Tevatron Upgrade provides for a an increase in duty factor to $50 \%$, this might be an acceptable approach. However, the currently evolving plans for a high intensity facility for neutrino experiments would conflict with this option.

If no new experiments are introduced in MEast, we could build new test beams that are fed from a common target (as in the old Meson Area), and extend downstream from the Meson Detector Building. This is a far more flexible solution than the NT option, but much more costly and dependant on the unlikely event that no new user will be found for the E-605 spectrometer magnet.

Because the neutrino program is no longer running, one might contemplate modifying the NC beam to feed test areas in addition to NWest. This would be similar in approach to the MEast plan mentioned above, but would require much more civil construction. This plan would also depend on the outcome of proposals being submitted for new neutrino experiments.

MT operates as a successful test beam facility and runs off a transmission target in the MWest beam line. This might serve as a model for adding new test beams. For example, a transmission target placed at the downstream end of either PWest or PB in enclosure $\mathrm{H}$ of the Proton Area could act as a production target for a new beam line that would extend about 1000 feet to a small new detector hall upstream of the Pagoda.

Another possibility would be to bring a new beam out of the Q-stub. This would involve adding a splitting station in the Switchyard, building a new targeting area, and the construction of beam lines and a detector hall for test beam activities. This might be feasible if limited to proton beam energies of $150 \mathrm{GeV}$. This facility might then operate off a front porch during fixed target runs.

\section{D.2. Nooks and Crannies}

There are several other possibilities for test beams in future runs depending on the kind of beam required. Muons are available in several locations: In the Muon Lab downstream of E-665, in Lab D downstream of E-690, and in MEast downstream of whoever will be running there. Electrons could be available in the Wide Band Hall when E-774 is complete and with a modest modification to the electron beam dump. 


\section{D.3. A Test Beam Facility At the $8 \mathrm{GeV}$ Booster}

There will always be a steady state low level need for low energy test beams. (a few $\mathrm{GeV} / \mathrm{c}$ down to a few hundred Mev/c) This is something the lab so far has never provided, but would be very convenient for testing chambers, silicon detectors, scintillators etc. Most of this work is now done with sources and cosmic rays, but if such a facility could be provided cheaply it would be desirable.

However, the Booster is not presently capable of delivering any sort of slow extracted beam, nor is there any way of implementing such a capability. The geometry of the ring is such that there is really no place to put any sort of an electrostatic septum which might be used to extract beam. Putting a wire into the Booster itself to scatter the beam slowly out should not be done because of radiation problems.

The only alternative for the Booster is to extract the beam in a single turn. The beam would come out at $8.9 \mathrm{GeV}$ in 84 bunches equally spaced over $1.6 \mu$ seconds. Such a beam can be extracted either into the AP4 line (towards the antiproton source) or into the $8 \mathrm{GeV}$ line (towards the Main ring). The lowest intensity currently accelerated is $5 \times 10^{9}$ protons/bunch $\left(4 \times 10^{11}\right.$ total); This corresponds to a single turn injection from the Linac. If the Linac is turned down, the Booster could still accelerate beam down to the level of about $1 \times 10^{9}$ protons per bunch. A reduction of this intensity to one particle / $\mu \mathrm{sec}$, as might be appropriate for a test beam, would require one proton for every 50 bunches, an attenuation of about $10^{11}$ over the minimum intensity the machine could provide.

There currently exists a beam dump in the AP4 line which kills essentially all of the Booster beam directed into it. It is likely that a few protons are dribbling out the back. One might be able to construct a line to capture these and send them into a facility west of the antiproton source. This would obviously be a speculative effort, since we have no way of knowing the amount of trickle beam that leaves the dump.

In general, the Booster offers no opportunity for a test beam; We must look to the high energy extracted beams for satisfying the test beam need.

\section{D.4. The P-Bar Source as a Test Beam Facility}

A new test area might be considered to run concurrently with the pbar target, while the Source is accumulating pbars. The new beam would view the target at a large production angle with small acceptance. The questions that must to be resolved include the duty factor, cost, and whether construction could take place without interrupting the running schedule. Unfortunately, the prospects are not bright for a facility of this sort.

The pbar source takes 1.5 microseconds of beam every 2.5 seconds from the main ring with intensities of about $10^{* *} 13 \mathrm{ppp}$. The targeting energy is ideal for many test beam requirements, but the duty factor is not useful for test beams. When the source is in operation, the repetition rate is limited by the heating effects of the target and focusing system.

Installation of a slow extraction system at $\mathrm{F} 17$ is possible, but would be of no value to the Source. Slow spill turns off the p-bar source. A slow extraction system to the fixed target areas would have much greater utility. 
The tunnel at F17 is crowded; Another beam line wouldn't fit. The installation of a secondary test beam there would turn off the Source during civil construction.

It's likely that operating a test beam at F17 would interfere with the operation of the Source. The Source is in operation all of the time: filling the Tevatron during collider runs, and providing p-bars for E-760 during fixed target runs.

For these reasons, F17 is not a good place to try to locate a test beam area.

\section{D.5. Test Beams at the New Injector}

A preliminary study was carried out for providing test beams at the site of the new injector. The beams would be extracted to a targeting area near the injector. Using one target, several beams could be brought out with different characteristics. For example, the area could contain a low energy beam ( 5 to $25 \mathrm{GeV})$, an intermediate energy beam (20 to $100 \mathrm{GeV})$, and a pure $120 \mathrm{GeV}$ proton beam. 
Appendix E

The SLAC and BNL Test Beam Programs

The Committee has looked into the availability of test beam at other laboratories:

Lew Keller at SLAC gave us a picture of test beam utilization there. Test beams have not run there for 2.5 years due to the effort being put into the SLC. Prior to this period, many Fermilab users took advantage of the SLAC external beams to carry out test beam work, especially on lead glass calibration and testing.

SLAC has four external beams that can be used as test beams. However, beam cannot be brought out to the external area while the SLC is operating. Some thought has been put into developing an extraction facility to take a few pulses when SLC is running, but this development program will be on hold until the SLC is fully operational. Even so, it isn't clear that extraction from the Linac can be accomplished without disturbing the beams in the SLC. There is therefore little hope for year round test beams at SLAC in the near future.

SLAC has a strong commitment to a nuclear physics program- N.P.A.S. - that will run for four weeks in FY89 and six weeks per year thereafter. Electrons can be extracted to the test beam areas when the nuclear science program is running, although the energy is limited to six GeV. The low energy renders the hadron beams unusable, but the electron beams are good. Nevertheless, there is likely to be little test beam work in FY89, because everyone at SLAC will be concentrating on the SLC effort.

Derek Lowenstein and Don Lazarus are the contacts at BNL. They have one test beam currently available - all other spigots are used by approved experiments; They assign space in the beam on a first come first served basis; Everyone is welcome to make use of the beam; The users define their own running schedule. "The AGS assumes as much of a laisser faire policy as possible when it comes to test beam use."

The Particle Detector Group's experience with test beams at other laboratories can be summarized as follows:

BNL has one high energy test beam (6 GeV p's and pions). It runs $16-20$ weeks per year and there are 6-8 groups on it at any one time.

SLAC has no beam available right now.

CERN has a dedicated beam line for each LEP experiment. There is only one non LEP beam line, and it is oversubscribed. There is little chance that anyone not involved in the CERN program could get any time on it.

Los Alamos has a high intensity line that gives $800 \mathrm{MeV}$ protons, pions and muons. This energy, like that of SLAC and BNL is a bit low for many Fermilab test beam needs. 
Appendix $\mathrm{F}$

Additional Comments

From: Henry Lubatti

Fermilab test beams and the SSC

I believe there will be great pressure on Fermilab to provide test beams for the SSC. The demand will be not only for testing new detector ideas but also for testing prototypes and tests of assembled modules (for example calorimeter modules). As I view it, one may expect enormous demands on Fermilab even though the SSC is not sited at Fermilab. I believe that each detector will require its own permanent test beam line. This is in fact what has happened at CERN with the LEP detectors. Further, these lines will require the capability of delivering hadrons over a range of energies and also electrons. But perhaps most significant is that given the sophistication of SSC detectors, these tests will require a rather sophisticated data acquisition system. Because of the size of the SSC detector components, crane coverage and good rigging facilities will probably be necessary.

At the SSC each approved detector will require its own permanent test beam. The issue is whether or not such test beams can be provided in a timely fashion at the SSC site. It is my belief that it will be very difficult to meet the requirements at the SSC site. The tests which will be carried out, as I have mentioned above, are sophisticated, complex tests which will require good data acquisition systems and good computer systems for analyzing the results. It is difficult for me to imagine that the SSC laboratory, which will be preoccupied with building an accelerator and much of the required infrastructure, will be able to provide ab initio the test beams and support which is necessary. Thus, the only laboratory which has the existing infra- structure and the range of possibilities that SSC detector test beams will require is and will continue to be Fermilab. I can well imagine that it will become necessary for Fermilab to provide dedicated test beams for some if not all of the SSC detectors. There is much logic to this as it will provide the SSC detector builders with the resident expertise and support of the Fermilab group. How this is interleaved with the main goal of Fermilab to provide research facilities during and beyond the SSC construction phase will have to be delicately worked out, but it seems inconceivable to me that Fermilab could refuse to provide test beams for SSC detectors. Thus I think it is crucial that the Fermilab management develop a scenario in concert with the SSC management of how to best provide these test beam facilities. Clearly the funding for the test beams must come from the SSC budget but I don't believe that will be the most serious problem. 